Cinémas

Revue d'études cinématographiques

Journal of Film Studies

\title{
De la synthèse au synthétique. Histoire de transparence
}

\section{Michel Arsenault}

Volume 7, numéro 3, printemps 1997

Cinéma québécois et États-Unis

URI : https://id.erudit.org/iderudit/1000953ar

DOI : https://doi.org/10.7202/1000953ar

Aller au sommaire du numéro

Éditeur(s)

Cinémas

ISSN

1181-6945 (imprimé)

1705-6500 (numérique)

Découvrir la revue

Citer cet article

Arsenault, M. (1997). De la synthèse au synthétique. Histoire de transparence. Cinémas, 7(3), 147-158. https://doi.org/10.7202/1000953ar

\section{Résumé de l'article}

L'image, qu'on disait hier de synthèse, devient aujourd'hui de plus en plus synthétique. Dans ce passage du visuel de la synthèse lisse et pure au synthétique rugueux et texture, la technologie atteint un nouveau seuil de transparence. Encouragée par le star system hollywoodien, elle infiltre chaque jour un peu plus l'espace écranique québécois. Mais, malgré ce danger culturel, l'identité québécoise trouve quelquefois des solutions de résistance originales. L'étude du film Screamers de Christian Duguay (1996) démontre que le cinéma québécois peut jouer la carte de la transparence hollywoodienne tout en s’opposant à son idéologie. 


\title{
De la synthèse au synthétique. Histoire de transparence
}

\section{Michel Arsenault}

\begin{abstract}
RÉSUMÉ
L'image, qu'on disait hier de synthèse, devient aujourd'hui de plus en plus synthétique. Dans ce passage du visuel de la synthèse lisse et pure au synthétique rugueux et texturé, la technologie atteint un nouveau seuil de transparence. Encouragée par le star system hollywoodien, elle infiltre chaque jour un peu plus l'espace écranique québécois. Mais, malgré ce danger culturel, l'identité québécoise trouve quelquefois des solutions de résistance originales. Létude du film Screamers de Christian Duguay (1996) démontre que le cinéma québécois peut jouer la carte de la transparence hollywoodienne tout en s'opposant à son idéologie.
\end{abstract}

\section{ABSTRACT}

In the present era of the visual, the image that was once a synthesis is today more and more synthetic. In moving from the smoothness and purity of synthesis to the coarseness and texturedness of the synthetic, technology has reached a new level of transparency. Driven by the Hollywood star system, it occupies an everincreasing share of Quebec screen space. Despite the cultural danger of American control over its film indusry, Quebecois identity sometimes finds novel solutions for self-affirmation. Studying the film Screamers by Christian Duguay (1996) shows how Quebec cinema can play Hollywood's transparency game, yet still oppose the values that underlie it.

Un verre d'eau sur le tableau de bord d'une voiture au cœur d'une forêt tropicale; un bruit plus sensible qu'audible nous 
prévient qu'en hors-champ, une menace gagne en importance. Sur la surface liquide, une onde donne corps à la sensation et suggère un frisson; plus encore, elle matérialise l'invisibilité à la frontière du son et des émotions. Le changement technologique adopte les traits d'un dinosaure virtuel. En observant de près cette créature made in Quebec, je ne peux m'empêcher de voir l'icône parfaite, parce que magistralement concentrée et concrétisée, de l'écrasant triomphe de l'esthétique hollywoodienne sur tous les cinémas du monde. En ce sens, quelques cerveaux québécois auront servi à nos voisins du Sud un produit capitaliste idéal: une bête aussi évanescente que la fluctuation des valeurs mobilières, un monstre aussi volatile que lucratif. Devant ce fait, n'est-il pas plus judicieux de considérer cette révolution technologique comme un profond mouvement réactionnaire et uniformisant dissimulé sous le spectacle? Plus spécifiquement, les réalisateurs du Québec et d'ailleurs sauront-ils mettre à profit cette expertise développée au Québec et explorer les possibles du virtuel sans se rendre prisonniers du vraisemblable hollywoodien?

Aspirés par la transparence du cinéma commercial étatsunien, nous vivons en ce moment le passage de l'image de synthèse à l'image synthétique. Sur les écrans contrôlés par les Majors, le film Forrest Gump (Robert Zemeckis, 1994) nous offre ces images générées par ordinateur qui ne présentent plus la moindre trace de synthèse; elles sont passées au synthétique, elles marient maintenant, dans l'invisibilité la plus complète, le documentaire et la fiction au sein d'une même composition écranique. Les réalisateurs hollywoodiens n'ont plus besoin de chercher le raccord invisible d'un plan documentaire à un plan fictif puisque dorénavant, les technologies numériques permettent leur coexistence dans un même champ. Si l'idéologie, comme le mythe, est une réorganisation des résidus de la réalité, ne peut-elle pas devenir, grâce à l'image synthétique, parfaitement transparente au réel ? L'image synthétique s'impose aujourd'hui des déformations, elle devient rugueuse, parasitée et imparfaite, elle tente de rejoindre la réalité. Elle ne craint pas de diminuer sa nature superficielle en renonçant, en apparence, au "[...] contrôle strict et entier des différents processus permet- 
tant de [se] générer [...]" (de Mèredieu, p. 6). Elle vise ainsi un contrôle plus profond et plus sournois. Dans Forrest Gump, le réalisateur Zemeckis fait se rencontrer un personnage de la fiction, Gump, et un personnage de la réalité, Kennedy, dans l'espace d'un même plan. Le spectateur assiste alors à la présentation d'une réalité synthétique. Lorsque le personnage fictionnel de Gump serre la main de Kennedy, il gagne en vraisemblance. L'invisibilité du montage effectue là un saut "quantique" vers la transparence parfaite d'une manipulation idéologique.

C'est à cette fin que la machine hollywoodienne désire fabriquer des images toujours plus vraisemblables. Les producteurs hollywoodiens utilisent les techniques de l'image synthétique pour accroître le réalisme de la mise en scène, son caractère vraisemblable. Mais l'expression "mise en scène" ne convient pas au cinéma états-unien commercial, il faudrait plutôt parler de reconstitution ou de synthèse du réel, car l'appellation de mise en scène admet au point de départ la notion d'auteur, elle suppose que la vision personnelle d'un artiste prenne forme dans une œuvre au détriment quelquefois du vraisemblable. En terre états-unienne, véritable "planète-réalité" où l'on doit non pas toucher, mais voir pour croire, l'idée même d'un cinéma d'auteur irrite toute l'industrie du cinéma. L'idéologie profonde, forgée depuis la déclaration d'Indépendance, fonde le visuel comme preuve d'existence légale: «[...] ce qui n'est pas visualisable n'existe pas" (p. 393), comme le dit Régis Debray de la société occidentale actuelle. Autrement dit, même en pays puritain, la justice n'est plus une question divine, mais une affaire visuelle. Par le biais de la caméra, c’est la réalité qui se fait justice elle-même. Ainsi, tout ce qui ne s'affiche pas à l'écran cathodique n'appartient pas au monde et à sa réalité, celle du visualisable. Nous nous retrouvons face à une télévision et à un cinéma de la fermeture. Contrairement à l'" œuvre ouverte" d'Eco, tout est joué d'avance et le montage chronologique se veut implacable: il n'existe rien au-delà ou en deçà de ce qui peut faire réagir le ruban magnétique ou la pellicule cinématographique. Par extension, le film tend à se valoir luimême, à se résoudre par lui-même. Les films hollywoodiens ne posent aucune question ni ne répondent à aucune venue de 
l'extérieur, ils ont la valeur immuable de ce qui est arrivé. Le plan lumineux de l'écran obtient le statut d'événement. Ici, contrairement au roman de Kundera, "la vie n'est pas ailleurs "... À l'ère du visuel, la vie d'un phénomène, d'une chose, d'un sentiment ou d'une personne n'est vraie que si elle est apparaît à l'écran (Debray, 1992).

Le cinéma et la télévision aux États-Unis, et souvent au Québec, se donnent à lire comme l'instant en action, niant l'opérateur de sens, la mise en forme et l'interprétance: «[...] l'autorité du réel immédiat favorise en somme l'escamotage des médiations (à la fois techniques, psychologiques, idéologiques, politiques, etc.) et accrédite ce mensonge naturaliste: la vision sans regard, ou la scène sans mise en scène" (Debray, p. 376). En Amérique du Nord, un homme est jugé davantage sur ce qu'il est capable de répondre instantanément à une question (donc en direct lors d'une entrevue à la télévision) que sur le fruit de sa réflexion fondamentale, dans son matériau propre (écriture pour l'écrivain, danse pour le chorégraphe, tableau pour le peintre, etc.).

En prenant appui sur le signe triadique de C. S. Peirce défini comme " $[\ldots]$ quelque chose qui tient lieu pour quelqu'un de quelque chose [d'autre] sous quelque rapport ou à quelque titre" (p. 121), je propose d'expliquer l'omniprésence du "direct " dans la médiation télévisuelle et cinématographique nordaméricaine. Je pense que l'informel du direct tend à faire de la structure triadique, propre à toute manifestation sémiotique', une simple monade qui, ne représentant plus un objet sous un rapport conceptuel, "présente" l'objet de la réalité. C'est que l'information (non seulement celle véhiculée par les bulletins de nouvelles, mais celle en un sens plus structurel, plus théorique ${ }^{2}$ ) tend à nier l'opération effectuée sur la forme par le diffuseur et toute son équipe; ce qui provoque, de l'autre côté du champ, le "gommage" symétrique de l'acte d'interprétation. La déresponsabilisation du diffuseur, qui prétend ne pas intervenir sur la réalité et ne pas utiliser des formes travaillées donc réfléchies, entraîne une démobilisation du spectateur qui ne peut qu'être témoin de l'événement, non plus re-présenté mais présenté, donc définitif, immuable et complet en soi. En fait, la macro- 
structure espère que le plus grand nombre de spectateurs tombe dans cette réaction béate devant le petit ou le grand écran, sans jamais que la forme ne soit analysée ou remise en question.

Concernant l'information, notons l'effort consacré à contextualiser une absence de forme, pour que, par le contact télévisuel ou cinématographique, le spectateur obtienne l'impression de toucher le monde sans médiation. D'autre part, soulignons aussi les rapports très étroits que l' "informel " entretient avec le genre documentaire par son apparente objectivité. C'est ce lien particulier information-documentaire qui s'avère de première importance dans le cheminement de ma réflexion sur les technologies de l'image synthétique parce qu'il engendre une triple adéquation: le documentaire, dans un jeu informel des matières de l'expression, "présente" sans représentation l'objet réel.

C'est ce "couple" triadique, documentaire/informel/objetréel, que récupère la cinématographie hollywoodienne pour jouer encore plus invisiblement avec les techniques de la transparence idéologique. Le prétexte de l'information documentaire devient la couverture parfaite pour arriver à la manipulation du spectateur. L'adéquation documentaire/informel/objet-réel pousse son ancrage tellement loin dans la culture que le spectateur moyen passe tautologiquement "du premier au troisième". Ainsi, dans Jurassic Park de Spielberg (1993), tous les efforts déployés pour faire de l'apparition des dinosaures une image crédible sont orchestrés autour de cette adéquation triadique. En cherchant à donner au spectateur un contenu apparemment pur, Spielberg devient journaliste: il inscrit dans son film les propos de divers spécialistes, il présente un documentaire de vulgarisation scientifique et termine son "topo" en nous "présentant" les monstres de jadis. Échappant à la représentation, ils sont comme nature. René Passeron dit de la présentation que c'est l'" [...] art de travestir ce qui se donne pour l'objet d'une révélation" (p. 14). C'est de cette manière que nous apparaissent les premiers dinosaures jurassiques, ils surgissent sous le mode de la révélation. Plus besoin de formes pour remplacer les objets, nous sommes maintenant face à eux sans médiation. Devant un écran à deux dimensions, nous faisons directement l'expérience du monde... 
Les cadrages de même que les mouvements de caméra frappent par leur fonctionnalité: ils sont condamnés à la "présentation " du monde jurassique comme, dans un studio des actualités télévisuelles, les caméras semblent se plier aux moindres caprices de la réalité. Ainsi, à l'écran cathodique comme à l'écran lumineux, plus de mise en forme, plus de représentation, tout ce qui doit être vu se voit dans le cadre: l'image n'a plus de profondeur. "L'image meurt, vive le visuel " scanderait avec ironie Régis Debray. Dans Jurassic Park, non seulement les images n'ont-elles plus de profondeur, mais plus encore, elles n'y trouvent plus leur forme propre; les images de Spielberg ressemblent à n'importe quelles images (au contraire, celles de Fellini, de Godard, de Welles, d'Arcand, d'Egoyan sont uniques). Elles basculent dans le cliché, le lieu commun. Si la technologie que nous développons sert le but états-unien, c'est-à-dire tout donner à l'œil pour tendre à effacer la présence de l'" acte sémiotique", nous devons nous montrer très prudent vis-à-vis de l'utilisation de ce nouvel outil et, autant que possible, en demeurer les maîtres.

Un récent film québécois utilise les techniques à la base de la production des images synthétiques: Screamers de Christian Duguay (1996). Les technologies de l'image synthétique ont permis à un réalisateur québécois de réaliser un film de sciencefiction qui n'a visuellement rien à envier à un film hollywoodien. Au strict niveau des effets spéciaux, les scènes construites par l'imagerie synthétique sont d'une grande réussite. Il n'y aurait à émettre qu'une seule réserve à propos des accessoires futuristes utilisés (vêtements, armes, déphaseurs, cellulaire, etc.) et à travers lesquels l'origine québécoise du film se devine le plus. Ici, la transparence se voile: malgré les modifications apportées, nous reconnaissons toujours le casque de hockey, le téléphone portatif, etc.

Sur le plan diégétique, ce film demeure plus complexe que les films commerciaux ordinairement issus des Majors. D'une part, le cynisme omniprésent du personnage principal envers le caractère manichéen des valeurs états-uniennes fondamentales jette un doute sur l'opposition franche entre le Bien et le Mal que représente l'affrontement entre le groupe auquel il appartient (représentant le Bien) et le Nouveau Bloc économique (incar- 
nant le Mal). Au bout du compte, ce seront les objets que l'homme avait si ardemment conçus et désirés qui deviendront porteur du mal. À ce titre, Screamers peut être interprété comme une métaphore du monde matérialiste dans lequel nous vivons. Il forge une hypothèse sur le futur (un futur déjà en formation dans le présent) de chaque individu soumis aux machines de visions capables de dévorer son identité et de lui voler son apparence. Revoyons ici l'évolution des "screamers" afin de mieux apprécier le travail sur la forme effectué par Duguay.

Au début du film, les armes biomécaniques (alternative intéressante à l'arme biologique) que sont les "screamers" ressemblent plus particulièrement à de cruelles scies radiales. Mais, à mesure que progresse le long métrage, Duguay nous présente différents types de "screamers" toujours plus humains, toujours plus ressemblants à l'être humain. La scie radiale devient un jeune enfant, l'enfant un soldat plus humain que l'humain. Le "screamer" est en quelque sorte l'incarnation du matérialisme de la société occidentale. Il adopte l'idéologie capitaliste de la mise en marché: comme l'objet de consommation, il se modifie pour adoucir ses formes et séduire toujours davantage sa "proie". La scène d'ouverture du film laisse aussi entendre que les "screamers" se livrent à un examen intensif du soldat: de toutes parts, ce dernier est ciblé par un œil objectif, il est analysé par un ensemble de curseurs et de viseurs pour être finalement démembré littéralement. Cette observation analytique permet aux "screamers» de disséquer le soldat du Nouveau Bloc économique (NEB), ils le dépècent pour mieux le connaître, cherchant la voie d'une absorption plus facile de l'humain. Le "screamer" sait que l'enveloppe visuelle de surface n'est pas garante de tout; c'est là une leçon que les personnages du film, à l'image de ceux de la réalité, dirait Debray, ont du mal à assimiler.

Pour les besoins du suspense, mais aussi pour proposer la vision du réalisateur, les monstres trament sous la surface, d'abord au sens propre, ensuite au sens figuré. Les "screamers" doivent d'abord demeurer sous le sable, ils possèdent tous les attributs de la machine. Mais à la suite de mutations, les «screamers" finissent par vivre au milieu des humains, la différence fondamentale entre les deux groupes n'existant plus. Les 
"screamers " parviennent maintenant à saigner, à aimer et finalement à contrôler leur apparence selon les exigences de la situation (l'ourson auquel le personnage principal s'était confié devient le dernier "monstre" du film). La dangereuse conséquence de cette progression, c'est que les monstres n'auront plus jamais une "sale gueule": la machine de guerre brute s'est modifiée au point de parvenir à s'immiscer dans la vie des personnages de la fiction sous l'apparence de la normalité. Les "screamers" sont parvenus à une telle réussite que l'humain même ne supporte plus la comparaison. Le soldat du NEB est assassiné par le personnage interprété par Roy Dupuis (un véritable "screamer", celui-là) parce qu'il n'avait pas un vocabulaire suffisamment considérable pour être un véritable humain.

Une comparaison s'impose d'elle-même entre l'évolution des machines de guerre et l'idéologie à la base du cinéma hollywoodien. Dans Screamers, la technologie devient de plus en plus discrète, les objets de guerre se camouflent, se moulent et s'enfouissent sous les apparences. Tout cela finit par ressembler au paroxysme de la transparence. Pourtant, la transparence s'y trouve mise en procès, non par la technique fondamentale, mais par la diégèse et le travail iconique autour de la proposition de l'incarnation du $\mathrm{mal}^{3}$. Si Screamers respecte la règle générale d'invisibilité du cinéma hollywoodien, il n'a pas pour autant recours à toutes les techniques de l'invisibilité telles que définies par Paul Warren (1989): le montage parallèle et le coulissage entre le off et le in sont absents de ce film. Autrement dit, la transparence devient l'affaire d'un système complexe refermé sur lui-même, où une seule et unique ligne diégétique se construit en transparence sur le raccord de mouvement (ou de regard) et l'ellipse spatio-temporelle. L'incidence la plus importante sur l'identité québécoise de ce film provient de l'absence de montage parallèle qui implique à son tour l'absence du reaction shot pur ${ }^{4}$. Warren affirme en analysant Le Triomphe de la volonté de Leni Riefenstahl que "[...] le reaction shot écranique aurait donc affaire au réalisme" $(1989$, p. 13). C'est en ce sens que je prolonge sa définition du reaction shot en proposant que cette technique "présente" des personnages ayant le même "statut" que les spectateurs dans la salle. L'absence de reaction shots purs dans 
Screamers implique donc une conséquence de taille: ce film ne s'approprie pas la réalité, il ne se ferme pas invisiblement en enchâssant le documentaire dans la fiction. Il se ferme ostensiblement pour signifier qu'il ne présente pas une réalité vraisemblable, mais qu'il "représente" plutôt l'hypothèse, l'abduction ", la vision particulière d'un artiste. Voilà pourquoi j'irai jusqu'à dire que le film de Duguay fait le procès de la transparence hollywoodienne en créant des monstres à la monstruosité invisible.

Comme je l'ai mentionné précédemment, le cinéma hollywoodien préconise la présentation plus que la représentation parce que la représentation force à " [...] mettre en relation, en présence, au sein de la représentation, l'acteur et le spectateur [...] (Chabert, p. 201), distanciation que les Majors veulent à tout prix éviter et contre laquelle ils développent le star system et son pendant obligé, le reaction shot. La présentation suppose "[...] la manifestation extérieure du caractère, sa révélation, sa projection" (p. 193), elle tend à se donner comme une vérité absolument objective et totalement visible. Dans Screamers, la mise en scène manifeste justement un rejet ostensible des valeurs et de la transparence états-uniennes. Certes superficiellement, Screamers fait toujours raccord dans la continuité et le montage, mais, sous la surface, l'absence d'une volonté d'intégration du hors-champ (par la voix off ou le montage parallèle) affirme un cinéma différent, un cinéma qui donne le choix. Le spectateur se voit confronté, dans son individualité, aux formes tantôt synthétiques, tantôt organiques, mais toujours virtuelles et mises à la place d'une vision. Jamais, dans le film, il ne trouvera sur l'écran lumineux un regard assimilant le sien, le complétant, lui apportant une réponse simple et sans équivoque sur cette vision inquiétante du rapport entre l'homme et ses machines.

La scène de la communication holographique avec un dirigeant de l'Alliance représente iconiquement l'enjeu du film entier. Pour les personnages du film, cette "présentation" de l'autre personnage n'en est pas une, dans la mesure où elle possède une valeur événementielle: elle a le même statut qu'une véritable rencontre. Pour une première fois dans le film, les personnages se font prendre au jeu des apparences; ce ne sera pas la dernière. Ce jeu des apparences est ici orchestré intradiégétique- 
ment par les technologies de l'image synthétique; il remet en scène le couple triadique documentaire/informel/objet-réel auquel spectateurs et personnages, chacun dans son monde, seront confrontés tout au long du film. Screamers propose donc une réflexion sur le bien-fondé de cette triple adéquation tautologique. En fait, il scande, à la manière des engins de la mort que sont les "screamers", cette méfiance en face de ce qui semble trop parfait, trop transparent, trop "présentifié".

Bien qu'il faille regretter, dans Screamers, l'absence d'un développement de nouvelles formes grâce à l'image synthétique et l'utilisation principalement illustrative, documentaire et transparente des nouvelles technologies de l'image, il n'en reste pas moins que la fermeture du système de représentation permet d'enrayer la mort de l'âme et de l'image, et fait de l'invisibilité le véritable objet du long métrage.

En conclusion, je salue ce tour de force réalisé par Duguay qui retourne contre lui-même le système hollywoodien. Un tour de force d'autant plus grand qu'il s'effectue malgré la lourde bureaucratie qu'entraîne un budget de 14 millions \$ au Québec. Malgré toutes les embûches et responsabilités qu'une telle somme présente, Duguay est parvenu à réaliser "son" film, un film véritablement maîtrisé. C'est qu'un danger plus grand que celui des technologies de l'image synthétique menace le cinéma et les metteurs en scène québécois: l'industrie semble vouloir, elle aussi, jouer la carte de la triple adéquation. Les structures de l'industrie cinématographique québécoise sont calquées sur celle du système hollywoodien alors que le marché auquel elle s'adresse ne représente qu'une fraction de celui de sa voisine du sud.

En observant la production cinématographique québécoise des dernières années, il faut se demander si cette importation systématique - il n'y a pratiquement pas eu d'adaptation du système à la réalité québécoise - ne s'avère pas plus dangereuse que l'outil informatique. Dans un système pareil, la dimension des budgets devient telle que l'apport de l'État ne représente jamais assez et qu'il faut, comme les gens d'Hollywood, miser sur le succès commercial d'un film pour qu'il devienne profitable. Les réalisateurs doivent ainsi se soumettre aux impératifs des 
entrées en salles et construire leurs films en fonction des attentes d'un public moyen. Dans notre cas, ceci représente un défi complexe dans la mesure où notre marché souffre d'étroitesse. Inévitablement, le réalisateur québécois doit envisager d'élargir son espace de réception, complexifiant du coup sa définition du public moyen et de ses attentes.

En "mimant " le système hollywoodien, l'industrie cinématographique québécoise, même si elle est largement supportée par l'État, adopte implicitement l'idéologie états-unienne. Rapidement, les impératifs du marché et les obligations de rentabilité prennent le pas sur le développement d'une culture spécifique et originale. Ce choix politique a pour résultat la construction d'une image souvent standard et commerciale, copie conforme à l'esthétique hollywoodienne, une image qui n'en est presque plus une, tant elle paraît "objective». Si une image ne remplit plus sa fonction de représentation et de signification, comment peut-elle exprimer autre chose que les valeurs du capital et de l'argent?

La définition d'une culture originale et riche passe par l'image qui remplace, qui suggère ce qu'on ne peut voir. C'est en empruntant la voie de la représentation que le cinéma québécois (synthétique ou non) maintiendra son existence en marge de la transparence et de la "non-culture" états-unienne. En ce sens, un réseau structuré et parallèle aux mégaproductions de style hollywoodien assurerait la croissance régulière de la cinématographie québécoise au-delà de la course vers la plus grande transparence.

Université Laval

\section{NOTES}

1 La sémiotique peircéenne propose que tout ce qui peut être perçu comme un signe possède une structure triadique: (1) une forme représente (2) un objet conceptuel en ayant pour effet (3) de mettre en relation le premier et le deuxième dans un signe équivalent chez l'interprète.

2 Le regard posé par la théorie de l'information sur la communication, et la sémiotique par voie de conséquence, considère la sémiosis (évolution des signes dans la pensée et de par le monde) dans une perspective très limitée. On ne conserve ici qu'un rapport statistique dyadique, c'est-à-dire que la quantité d'information véhiculée se mesure en vertu de ce qui est arrivé par rapport à tout ce qui pouvait se produire. Autrement dit, l'information au sens plus familier comme au sens plus théorique, 
traite l'événement, donc le sans-forme, l'informel. Eco lui-même faisait remarquer que " [...] la valeur " information " ne doit pas être identifiée avec la notion communiquée" (La Structure absente. Introduction à la recherche sémiotique, Paris: Mercure de France, 1972, p. 46).

3 Je fais allusion à l'icône de plus en plus complexe que forme Duguay autour du "screamer ". D'abord, il n'est rien de plus qu'une vulgaire lame de scie radiale, par la suite, l'objet qu'il représente va se préciser par les formes plus évoluées d'une machine de guerre qui développe sa propre conscience lui permettant de jouer la carte des apparences pour exterminer.

4 Je rappelle que le reaction shot a été mis au point par les studios hollywoodiens pour pallier à l'absence des acteurs lors de la projection du film: ces derniers ne pouvant ajuster leur jeu pour obtenir l'effet escompté, on a astucieusement imaginé d'incorporer les spectateurs dans le film. J'entends donc par reaction shot pur un plan filmant des réactions d'individus extradiégétiques (on ne peut même pas dire d'eux qu'ils sont des personnages), c'est-à-dire de gens qui possèdent pratiquement le même statut que les spectateurs.

5 Jean Fisette définit l'abduction peircéenne comme étant " [...] une argumentation à rebours, allant du conséquent à l'antécédent. Elle opère des "sauts cognitifs" grâce à des choix interprétatifs audacieux, pouvant conduire à des connaissances nouvelles [...]" (Introduction à la sémiotique de C. S. Peirce, Montréal: XYZ éditeur, 1990 , p. 71).

\section{OUVRAGES CITÉS}

Chabert, Pierre. "Présentation / Représentation / Mise en scène ", dans René Passeron (direction), La Présentation. Paris : Éditions du CNRS, 1985.

Debray, Régis. Vie et mort de l'image. Une histoire du regard en Occident. Paris: Gallimard, 1992.

Eco, Umberto. Le Signe. Bruxelles: Éditions Labor, 1988.

Eco, Umberto. La Structure absente. Introduction à la recherche sémiotique. Paris: Mercure de France, 1972.

Fisette, Jean. Introduction à la sémiotique de C. S. Peirce. Montréal : XYZ éditeur, 1990.

Mèredieu, Florence de. "Le crustacé et la prothèse", Paysages virtuels. Paris: Éditions Dis Voir, 1988.

Passeron, René. "Création, présentation, présence", dans René Passeron (direction), La Présentation. Paris: Éditions du CNRS, 1985.

Peirce, Charles Sanders. Écrits sur le signe. Paris: Éditions du Seuil, 1978.

Warren, Paul. Le Secret du star system américain. Une stratégie du regard. Montréal: l'Hexagone, 1989. 